\title{
Health Effects of E-Cigarettes with or without Nicotine
}

\author{
Khaled Alawam*, Abdulwahab Alahmari, Alanoud Alhamed, Alia Alblowi, Atheer Alanazi, Hasan Alshagag, \\ Rawan Aenezi, Shaima Almasri, Suniya Altareef and Zainb Aldaqouq
}

Department of Respiratory Therapy Program, Inaya Medical College, Saudi Arabia

Submission: October 16, 2020; Published: November 11, 2020

*Corresponding author: Khaled A Alawam, Department of Respiratory Therapy Program, Inaya Medical Colleges, Riyadh 13322, Saudi Arabia

Keywords: Nicotine; Cigarette smoke; Pneumonia; Airway inflammation; Neutrophils

Abbreviation: SIDS: Sudden Infant Death Syndrome; COPD: Chronic Obstructive Pulmonary Disease; FeNO: Fractional Exhaled Nitric Oxide; PFTs: Pulmonary Function Tests; ECA: Electronic Cigarette Aerosol; TER: Transendothelial Electrical Resistance; NRT: Nicotine Replacement Therapy

\section{Introduction}

In Recent times, there is remarkable worldwide increase in the use of electronic nicotine products and also electronic nicotine delivery system [1]. Vaping is the act of inhaling and exhaling the aerosol, it refers as a vapor device, the term is used because e-cigarettes do not produce tobacco smoke, but rather than aerosol, often mistaken for water vapor, that actually consists of fine particles. Many of these particles contain varying amounts of toxic chemicals, which have been linked to cancer, as well as respiratory and heart disease. Vaporizer products usually contains of propylene glycol or vegetable glycerin-based liquid with nicotine, flavoring and other chemicals and metals, but not tobacco $[2,3]$.

E-cigarettes components can dry out mucous membranes and eyes, nicotine exposure can cause dizziness, nausea, vomiting, diminish coronary blood flow and impair endothelial function E-cigarettes have high risk of nicotine toxicity because of high concentration of nicotine in the cigarettes. Some studies reviewed the physiologic effects associated with acute exposure to E-cigarettes: Dry cough, mouth and throat irritation, increase in heart rate, and elevated WBC and decrease in fractional exhaled nitric oxide (FeNO). It was also seen that $\mathrm{E}$ - cig possess various risks of non-users: nicotine aerosols can stay on surfaces for weeks and months, children at high risk for toxicity from refill cartridges $[1,4,5]$. So, it was suggested to include "risk of toxicity in children" label mandatorily on E-cigarette liquids with warnings of other health effects [6].
The effect of smoke inhalation in cardiovascular system shows an increase the risk of developing atherosclerosis a disease which can cause heart attacks, strokes and can even lead to death [7].

Nicotine associated with increased cardiovascular mortality in virtually every cardiac population study, as well as in population without known cardiac disease [8]. Cigarette smoking is the primary causative for COPD, the third leading Cause of death [9]. E-cigarette users have levels of innate defense proteins associated with chronic obstructive pulmonary disease such as elastase and matrix metalloproteinase9. Sputum of cigarette smokers and e-cigarette users have shown an increase of proteases of neutrophil and epithelial origin known as inflammatory mediators it's a major contribute to chronic lung disease [10]. In asthma patient, the use of E-cigarette can lead to lipoid pneumonia [11].

A comparison investigated the effect of exposure to aerosolized nicotine-free and nicotine-containing e- cigarette fluid in mouse lungs and normal epithelial cell. They found that the key to inflammation is nicotine [12]. E-cigarettes expose users to a range of toxicants, carcinogens, and metal particulates, so even using nicotine-free e-liquid may carry health risks [13]. The use of e-cigarette free nicotine with vapor extract is the reason of activation neutrophils continues up to 6 hours and stimulate neutrophils shape [14].

Exposure of electronic cigarettes during pregnancy will affect lung development of the fetus, low birth weight, prematurity, 
intrauterine growth restriction, and increase the risk of sudden infant death syndrome (SIDS), because of nicotine exposer before delivery will decrease the respiratory respond to hypoxia [15]. A study was done in a small sample that uses E-cigarettes focusing on people which began vaping, their reasons why they using vaping, their understanding of the harm effects of vaping compared to smoking, and their opinions in wither if it's increases their likelihood of using smoking. all interviewees said that both vaping and smoking has very different levels of harm, none of the interviewees felt that the use of e-cigarettes had increased their likelihood of smoking, when it comes to how the interviewees begun using vaping it occurred in their social contacts with their friends in which they had been asked or offered to try [16].

Comparison was done between adolescents and adult using e-cigarette flavors, adults preferred a greater total number of flavors compared to adolescents and used e-cigarettes more frequently [17]. Electronic cigarettes are interpreted as a safer alternative compared to combustible cigarettes (c-cigarettes) $[18,19,20]$. Studies has been shown combination nicotine replacement therapy shows additive cessation benefits. The use of nicotine replacement therapy helps in reducing the symptoms associated with people want to quit smoking [21].

\section{Human Health Effects}

Most of the E-cigarettes consists of glycol and glycerol, which can dry out mucous membranes and eyes, nicotine exposure can cause dizziness, nausea, or vomiting E-cigarettes have high risk of nicotine toxicity because of high concentration of nicotine in the cigarettes. The physiologic effects associated with acute exposure to E-cigarettes: Dray cough, mouth and throat irritation, increase in heart rate, elevated WBC, and decrease in fractional exhaled nitric oxide (FeNO). Exposure risks for non-users: nicotine from the aerosol can stay on surfaces for weeks and months, Children's are at high risk for toxicity from refill cartridges [1].

The acute effect with high nicotine level and long duration on hemodynamic effect, with those of regular cigarettes smoking. Smoking inhalation increase inflammation, thrombosis and oxidation of low-density lipoprotein cholesterol that contributes to cardiovascular dysfunction. Use of electronic cigarettes had no impact on the exhaled CO levels, but there is significantly elevate the exhaled CO more than 8 times above the baseline with manufactured cigarette. Which is indication of higher risk in cardiovascular dysfunction for cigarette smokers [7].

Effect of the E-cigarettes on the airways, e-cigarette users have levels of innate defense proteins associated with chronic obstructive pulmonary disease such as elastase and matrix metalloproteinase 9. sputum of cigarette smokers and e-cigarette users have an increase of proteases of neutrophil and epithelial origin known as inflammatory mediators it's a major contributor to chronic lung disease [10]. E-cigarettes associated with flavoring agents may produce harmful effects in stem cells and gingival fibroblasts by generating aldehydes/carbonyls from electronic cigarette vapor while c-cigarette smoking worsens cardiac and renal function in humans and in animal models. An animal research was done shown that, short-term exposure to e-cigarette vaping does not have a significant effect on cardiac contractility and geometric properties, but it increases the angiogenesis represented by capillary density in mouse heart tissue [18].

Smoking during pregnancy is the largest cause of low birth weight, prematurity, intrauterine growth restriction, and perinatal mortality. Sadly, more than $50 \%$ of smokers continue to smoke during pregnancy. Smoking during pregnancy adversely affects fetal lung development, causing offspring to fail to reach maximum lung function in childhood with subsequent lifelong decreases in pulmonary function. Infants born to smokers show decreased pulmonary function tests (PFTs), with decreased respiratory flows and respiratory compliance and altered tidal breathing patterns, at birth and before any significant exposure to postnatal smoke. Studies in monkeys and mice have primarily been performed to show the effects of prenatal nicotine on lung development, exposure to prenatal nicotine alone, at levels similar to that of smokers, causes similar decreases in FEF. Finally, there is large link between smoking during pregnancy and the increased risk of offspring dying from sudden infant death syndrome (SIDS). The findings are strongly suggested that use of e-cigarettes during pregnancy will have the same effect on lung development and offspring lung health as does the use of conventional cigarettes [15].

Human studies found increased risk of respiratory symptoms, chronic bronchitis symptoms (chronic cough, phlegm, or bronchitis). Cross-sectional surveys have also found associations of e-cigarette use with a history of asthma and with asthma exacerbations. Also, there is a Study found that e-cigarette use was associated with higher self-ratings of dyspnea and reports of COPD and asthma. Significant pathologic changes in small airway/ alveolar destruction, have been observed in early stage smoking induced COPD despite relatively few changes to FEV1. A recent controlled study in healthy young occasional smokers showed that just 15 minutes of exposure to aerosol from an e-cigarette impaired gas exchange and reduced expiratory gas flows, these results suggest that e-cigarettes may cause acute, physiologically detectable injury to the small airways. In Animal studies found that the effects of e-cigarettes have been performed in mice, with durations of exposure ranging from a single dose to three to six months using propylene glycol/ vegetable glycerin with or without nicotine and commercial e-liquids. Acute exposures have caused changes at the level of the protein, including up-regulation of mucins and cytokines, as well as cellular changes including impaired autophagy. 
Chronic e-cigarette exposures induce airway inflammation, neutrophilia, airway remodeling, and emphysema. Increased sensitivity to methacholine was also observed. Both nicotine dependent and nicotine independent effects were found. Vaping in mice leads to rapid changes at the cell and protein levels. Of greater concern, five to 16 weeks of e-cigarette exposure induced alveolar cell apoptosis and architectural simplification suggestive of emphysema. Studies have been done in humans saying E-cigarettes are safer than combustible cigarettes are impossible. Given the survey data showing increased symptoms of respiratory disease and the many lines of human, animal, and in vitro experimental evidence that e-cigarette aerosol can negatively affect multiple aspects of lung cellular and organ physiology and immune function [22].

\section{Effects of Vaping with and without Nicotine}

Nicotine is a sympathomimetic drug that acts on nicotinic receptors located throughout the autonomic nervous system to increase sympathetic tone and catecholamine release. Therefore, it is not surprising that acute exposure to electronic cigarettes with nicotine increased cardiac sympathetic nerve activity as measured by heart rate variability [8].

Exposure to electronic cigarettes increased airway obstruction. Two studies found that inhalation of electronic cigarette aerosol (ECA) with nicotine caused an increased arterial stiffness that may lead to high risk for myocardial infarction and stroke. Other studies showed lower amounts of urinary biomarkers for oxidative stress and carcinogens in chronic e-cigarette users compared to smokers. ECA exposure will increased fractional exhaled nitric oxide (FeNO). Vascular and pulmonary measurements were performed. Nicotine may cause diminish coronary blood flow, impair endothelial function, inflammation, arteriogenesis, and insulin resistance. The acute vascular and respiratory effects of e-cigarette aerosol with and without added nicotine. All vascular measurements are shown that increase in systolic- diastolic blood pressure that remained elevated for 10 and $30 \mathrm{~min}$, and increased heart rate for 20 min compared to ECA without nicotine [5].

A cross-sectional survey was done about tobacco and electronic cigarettes use the analytic sample comprised pastmonth e-cigarette users and examined them whether age, sex, smoking status, electronic cigarettes use frequency, and/or e-cigarette acquisition source were associated with using nicotinefree e-liquid, nicotine e-liquid, or not knowing the e-liquid nicotine concentration. Most adolescents do not know the concentration level of nicotine in the e-cigarettes that they use. However, male adolescents do it more than their female counterparts do. Those of them who are smokers tend to use e-cigarettes with higher concentration of nicotine, which they purchase either online or from shops. Non-smokers on the other hand use e-cigarettes with lower concentrations of nicotine, which they purchase online, or from their friends. The fact that these adolescents are oblivious to the effects of nicotine in their health is a major concern. This is worsened by the fact that they are unaware of the concentration level of nicotine in these e-cigarettes [13].

Effects of exposure to aerosolized nicotine-free and nicotinecontaining e-cigarette fluid on mouse lungs and normal human airway epithelial cells. The e-cigarettes increase oxidative stress and inflammation in mouse, inhaled nicotine in e-cigarettes triggered effects of COPD the nicotine increase airway hyperreactivity, enlargements in airways and mucus production. Key of inflammation and enlargements of airways that can lead to COPD is nicotine [12]. E-cigarette increase the release of inflammation mediators from alveolar epithelial cell line, E-cigarette vapour extract is the reason of activation of neutrophils and stimulate neutrophils shape change [14].

Primary cause for chronic obstructive pulmonary disease (COPD) is cigarette smoking and it is a third cause of death in all society in the world. Comparison was done on the effect of dissolvable sample from nicotine-containing and nicotinefree cigarettes to confirm the assistance of nicotine in CS to waste lung endothelial barrier function. Notice big decreases in transendothelial electrical resistance (TER) at incubation of primary rat lung endothelial cells with increasing concentrations of nicotine. The results show that nicotine has pulmonary effects related to the dose, this result will loss of lung endothelial barrier function, acute lung inflammation, and decreased lung endothelial cell proliferation [9].

The effects of exposure of mice to components of vaporized e-cig liquids in acute and sub chronic condition on the respiratory functional parameters and inflammatory responses and to compare them with exposure to air and classic cigarette smoke (CS) by using animal model. Total BALF cell count was elevated only in the CS and nicotine with flavor groups, BALF protein content was elevated in the nicotine group and nicotine with flavor groups only [2]. Short-term effects of e-cigarettes on exhaled nitric oxide. The authors have observed that while using E-cigarettes both with and without nicotine can lead to an immediate reduction of exhaled nitric oxide in smokers. Also, there have been reported that glycerine-based oils from the E-cigarettes can cause lipoid pneumonia [4].

\section{Use of E-Cigarettes in Smoking Cessation}

Cessation from tobacco smoking is likely to reduce the risk of respiratory Infection, lung cancer and pneumonia. Electronic cigarettes are emerging as an attractive long-term alternative nicotine source to conventional cigarettes consumption. Switching from smoking to vaping is likely to produce lung health benefits. Fast innovation in the e-vapor category is likely not only to further minimize residual health risks, but also to maximize health benefits in regular EC users [3].

Nicotine replacement therapy (NRT) shows benefits in smoking cessation. The use of nicotine replacement therapy helps in reducing the symptoms associated with people want to quit 
smoking, e-cigarettes mostly with nicotine, are used by smokers in many countries as an aid to quit smoking. A study was done to find out the efficacy of nicotine patches with an e-cigarette without and with nicotine in smoking cessation. The study concluded that patches and nicotine e-cigarette showed improvement in smoking cessation over the use of nicotine patches only and patches plus nicotine-free e-cigarette [21].

\section{Conclusion}

The use of electronic cigarettes is rapidly increased worldwide among adults and adolescents, the main reasons of using E-cigarettes among people are curiosity, flavoring, and its low harmful effects compared to other tobacco products. However, use of E-cigarettes can cause harmful effects on human health. Vapour extract contains glycerol, by heating glycerol it produces acrolein, which is a constituent of tobacco smoke. Awareness on its harmful effects may be helpful in reducing the use of E-cigarettes. Research has been shown that use of E-cigarettes with nicotine or without nicotine can cause harmful effects on the human body. Further research should be done on the use of E-cigarettes in smoking cessation.

\section{References}

1. Callahan Lyon P (2014) Electronic cigarettes: human health effects. Tobacco control 23(suppl2): ii36-ii40.

2. Glynos C, Bibli SI, Katsaounou P, Pavlidou A, Magkou C, et al. (2018) Comparison of the effects of e-cigarette vapor with cigarette smoke on lung function and inflammation in mice. Am J Physiol Lung Cell Mol Physiol 315(5): L662-L672.)

3. Campagna D, Amaradio MD, Sands MF, Polosa R (2016) Respiratory infections and pneumonia: potential benefits of switching from smoking to vaping. Pneumonia 8: 4.

4. Marini S, Buonanno G, Stabile L, Ficco G (2014) Short-term effects of electronic and tobacco cigarettes on exhaled nitric oxide. Toxicol Appl Pharmacol 278(1): 9-15.

5. Antoniewicz L, Brynedal A, Hedman L, Lundback M, Bosson JA (2019) Acute Effects of Electronic Cigarette Inhalation on the Vasculature and the Conducting Airways. Cardiovasc Toxicol 19(5): 441-450.

6. Schober W, Szendrei K, Matzen W, Osiander Fuchs H, Heitmann D, et al. (2014) Use of electronic cigarettes (e-cigarettes) impairs indoor air quality and increases FeNO levels of e-cigarette consumers. Int J Hyg Environ Health 217(6): 628-637.

7. Yan XS, D Ruiz C (2015) Effects of using electronic cigarettes on nicotine delivery and cardiovascular function in comparison with regular cigarettes. Regul Toxicol Pharmacol 71(1): 24-34.
8. Moheimani RS, Bhetraratana M, Peters KM, Yang BK, Yin, F et al. (2017) Sympathomimetic effects of acute E-cigarette use: role of nicotine and non-nicotine constituents. J Am Heart Assoc 6(9): e006579.

9. Schweitzer KS, Chen SX, Law S, Van Demark M, Poirier C, et al. (2015) Endothelial disruptive proinflammatory effects of nicotine and e-cigarette vapor exposures. Am J Physiol Lung Cell Mol Physiol 309(2): L175-L187.

10. Reidel B, Radicioni G, Clapp PW, Ford AA, Abdelwahab S, et al. (2018) E-cigarette use causes a unique innate immune response in the lung, involving increased neutrophilic activation and altered mucin secretion. Am J Respir Crit Care Med 197(4): 492-501.

11. Lappas AS, Tzortzi AS, Konstantinidi EM, Teloniatis SI, Tzavara CK, et al. (2018) Short-term respiratory effects of e-cigarettes in healthy individuals and smokers with asthma. Respirology 23(3): 291-297.

12. Garcia Arcos I, Geraghty P, Baumlin N, Campos M, Dabo AJ, et al. (2016) Chronic electronic cigarette exposure in mice induces features of COPD in a nicotine-dependent manner. Thorax 71(12): 1119-1129.

13. Morean ME, Kong G, Cavallo DA, Camenga DR, Krishnan Sarin S (2016) Nicotine concentration of e-cigarettes used by adolescents. Drug Alcohol Depend 167: 224-227.

14. Higham A, Rattray NJ, Dewhurst JA, Trivedi DK, Fowler SJ, et al. (2016) Electronic cigarette exposure triggers neutrophil inflammatory responses. Respiratory research 17: 56

15. Spindel ER, Mc Evoy CT (2016) The role of nicotine in the effects of maternal smoking during pregnancy on lung development and childhood respiratory disease. Implications for dangers of e-cigarettes. Am J Respir Crit Care Med 193(5): 486-494.

16. Mc Keganey N, Barnard M, Russell C (2018) Vapers and vaping: e-cigarettes users views of vaping and smoking. Drugs: Education, Prevention and Policy 25(1): 13-20.

17. Pisinger C, Dossing M (2014) A systematic review of health effects of electronic cigarettes. Prev Med 69: 248-260.

18. Shi H, Fan X, Horton A, Haller ST, Kennedy DJ (2019) The Effect of Electronic-Cigarette Vaping on Cardiac Function and Angiogenesis in Mice. Scientific reports 9: 4085

19. Schripp T, Markewitz D, Uhde E, Salthammer T (2013) Does e-cigarette consumption cause passive vaping? Indoor air 23(1): 25-31.

20. Morean ME, Butler ER, Bold KW, Kong G, Camenga DR, et al. (2018) Preferring more e-cigarette flavors is associated with e-cigarette use frequency among adolescents but not adults. PloS one 13(1): e0189015.

21. Walker N, Parag V, Verbiest M, Laking G, Laugesen M, et al. (2019) Nicotine patches used in combination with e-cigarettes (with and without nicotine) for smoking cessation: a pragmatic, randomised trial. The Lancet Respiratory Medicine 8(1): 54-64.

22. Gotts JE, Jordt SE, Mc Connell R, Tarran R (2019) What are the respiratory effects of e-cigarettes? BMJ 366: 15275 . 
Your next submission with Juniper Publishers will reach you the below assets

- Quality Editorial service

- Swift Peer Review

- Reprints availability

- E-prints Service

- Manuscript Podcast for convenient understanding

- Global attainment for your research

- Manuscript accessibility in different formats ( Pdf, E-pub, Full Text, Audio)

- Unceasing customer service

Track the below URL for one-step submission https://juniperpublishers.com/online-submission.php 